\title{
Gonorrhoea notifications and nucleic acid amplification testing in a very low-prevalence Australian female population
}

\author{
In ... Australia \\ outside \\ of high- \\ prevalence \\ populations,
}

testing should

be minimised

or done by

culture unless

the pretest

probability is

high

Eric PF Chow PhD, MPH, MApplSc',

Glenda Fehler MSc, FMLT

Tim RH Read PhD, MBBS, FAChSHM ${ }^{12}$

Sepehr N Tabrizi $\mathrm{PhD}^{3}$

Jane S Hocking $\mathrm{PhD}, \mathrm{MPH}, \mathrm{MH}$ lthSC

Ian Denham MBBS, FRACP, FAChSHM

Catriona S Bradshaw MBBS(Hons), FACSHP, PhD

Marcus Y Chen MBBS, PhD, FAChSHM ${ }^{1,2}$

Christopher K Fairley PhD, FAFPHM, FACSHPI,

1 Melbourne Sexual Health Centre, Melbourne, VIC.

2 Monash University, Melbourne, VIC.

3 Royal Women's

Hospital Melbourne Melbourne, VIC.

4 University of Melbourne Melbourne, VIC.

echow@mshc.org.au

doi: 10.5694/mjal4.00780

Online first 30/03/15
$\mathrm{P}$ assive surveillance has shown a rapid increase in the number of gonorrhoea (Neisseria gonorrhoeae) notifications in Victoria, Australia, over the past decade. About $17 \%$ of all notified cases are in women, and of cases in men, $71 \%$ are among men who have sex with men (MSM).

Gonorrhoea notifications can occur from a positive culture or a nucleic acid amplification test (NAAT). NAATs are more sensitive than culture, particularly for urine sampling, ${ }^{2}$ which is the most common specimen type collected in Australia. However, NAATs for gonorrhoea are less specific than culture, and the specificity of NAAT varies by specimen type and testing platform, producing false-positive results that reduce the positive predictive value (PPV) when the prevalence of infection is low. ${ }^{3,4}$ The product information ${ }^{5}$ and United States sexually transmitted infection (STI) treatment guidelines warn against the use of NAAT in lowprevalence populations for this reason. ${ }^{6}$ In contrast, gonorrhoea culture has a specificity of $100 \%{ }^{7}$

A dual NAAT for the detection of chlamydia (Chlamydia trachomatis) and gonorrhoea infection was introduced in Australia in 2007, and is now substantially more commonly used than the single test for chlamydia detection. ${ }^{8.9}$ The Royal Australian College of General Practitioners screening guidelines recommend chlamydia screening for individuals aged 15-29 years, but only recommend gonorrhoea screening for individuals at higher risk. ${ }^{10}$ The prevalence of gonorrhoea is extremely low even among Australian women attending sexual health clinics $(0.3 \%-0.4 \%){ }^{11}$ Conversely, gonorrhoea prevalence is higher in certain populations, such as the Indigenous population in remote areas $(7.2 \%)^{12}$ and MSM $(7.1 \%){ }^{13}$ these individuals are advised to undergo gonorrhoea testing every 3-6 months.

\section{Abstract}

Objectives: To examine whether the rapid increase of gonorrhoea notifications in Victoria, Australia, identified by nucleic acid amplification test (NAAT) is supported by similar changes in diagnoses by culture, which has higher specificity, and to determine the proportion of tests positive among women tested.

Design, setting and participants: Retrospective analysis of Medicare reporting of dual NAATs in Victoria, Victorian Department of Health gonorrhoea notifications, and gonorrhoea culture data at the Melbourne Sexual Health Centre (MSHC), among women, 2008 to 2013.

Main outcome measures: Gonorrhoea notifications and testing methods. Results: Gonorrhoea cases identified by NAAT increased from 98 to 343 cases over the study period. Notifications by culture alone decreased from 19 to five cases. The proportion of NAATs positive for gonorrhoea in Victoria was low $(0.2 \%-0.3 \%)$ and did not change over time ( $P$ for trend, 0.66). Similarly, the proportion of women tested at the MSHC for gonorrhoea who tested positive $(0.4 \%-0.6 \%)$ did not change over time ( $P$ for trend, 0.70 ). Of untreated women who had a positive NAAT result for gonorrhoea and were referred to the MSHC, 10/25 were confirmed by culture.

Conclusions: The positivity of gonorrhoea in women identified by culture remains stable over time. Using NAAT for gonorrhoea screening in lowprevalence populations will result in many false positives. Positive NAAT results among low-risk women should be regarded as doubtful, and confirmatory cultures should be performed.

If NAAT is widely used among Australian women with low gonorrhoea prevalence, the low PPV of NAAT may cause substantial harm. ${ }^{3,14}$ We therefore aimed to evaluate the proportion of positive NAATs among women, and whether this changed over time. We also assessed possible changes in gonorrhoea prevalence by analysing women tested by culture alone in a sentinel sexual health clinic population.

\section{Methods}

We performed a retrospective analysis of data from three sources: Medicare reporting of dual NAAT detection and gonorrhoea notifications for Victoria, and gonorrhoea culture at the Melbourne Sexual Health Centre (MSHC), among women, from 1 January 2008 to 31 December 2013. This period was chosen to study the effect of the introduction of the dual NAAT in May 2007 in Australia.
The study received ethics approval from the Alfred Health Human Ethics Committee (no. 147/14).

\section{Data sources}

We reviewed Medicare Item Reports from Medicare Australia for chlamydia testing alone (Medicare Benefits Schedule [MBS] item 69316) and the dual NAAT (MBS items 69317 and 69319) for chlamydia and a second or third organism (which predominantly reflects the multiplexed assay for chlamydia and gonorrhoea) among women in Victoria during the study period. ${ }^{15}$

As gonorrhoea is a notifiable disease in Australia, the annual notified cases among women, stratified by test type (ie, NAAT alone, culture alone or NAAT and culture), were obtained from the Victorian Department of Health.

We also reviewed the electronic database records of all women attending the MSHC over the same period to determine if changes had occurred at the MSHC, which uses culture only for 
1 NAATs for chlamydia only and chlamydia with another infection ordered through Medicare Australia among women in Victoria, 2008-2013

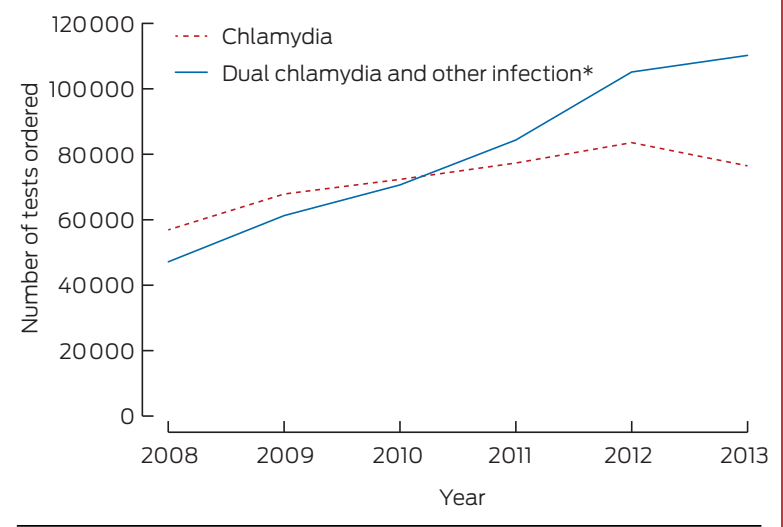

NAAT $=$ nucleic acid amplification test. $*$ Other infection is largely gonorrhoea.

2 Gonorrhoea notifications among women in Victoria, 2008-2013,* by year and types of laboratory tests

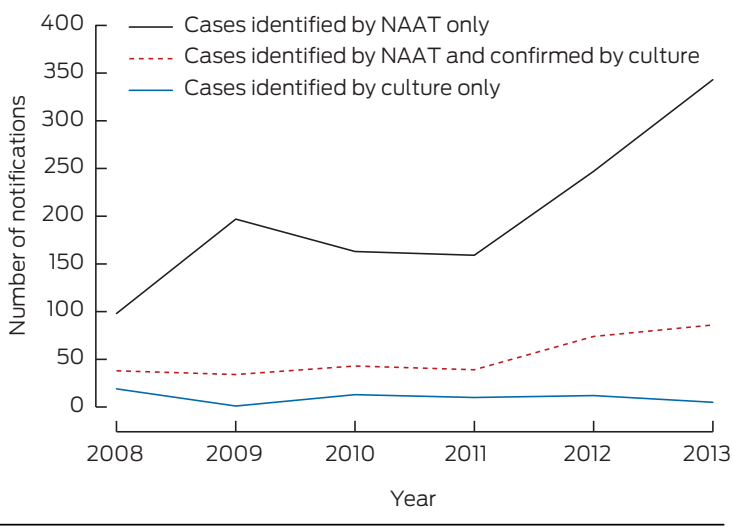

NAAT = nucleic acid amplification test. $*$ Cases tested by culture at Melbourne Sexual Health Centre are excluded.

gonorrhoea detection. The MSHC is the largest public sexual health centre in Victoria. Patients' demographic characteristics, sexual behavioural data and clinical diagnoses were collected and recorded in the electronic medical system at each clinic visit. All women who attended the MSHC during the study period were included in the analysis and the data were de-identified.

Women who presented to the MSHC with symptoms of an STI (eg, vaginal discharge), those at higher risk (eg, Indigenous people), those who had engaged in high-risk behaviour (eg, overseas contacts, injecting drug use, and contact with gonorrhoea) and sex workers were offered testing for gonorrhoea. Consenting individuals had endocervical swabs taken for culture by a clinician. All swabs were directly plated onto gonococci agar in the clinic room and immediately delivered to the onsite laboratory for culture. We also reviewed the records of women who were referred by GPs to the MSHC with a positive NAAT result of gonorrhoea who had not had treatment and had a swab taken for gonorrhoea culture at the MSHC.

\section{Statistical analysis}

Descriptive statistics and frequency distribution of the sample were calculated. The percentage of women who tested positive for gonorrhoea over the study period was calculated. A $\chi^{2}$ test for trend was used to evaluate the significance of gonorrhoea positivity in women over time. Data were analysed using SPSS, version 21.0 (SPSS Inc).

\section{Results}

There was a 2.3-fold increase in the use of dual NAATs for chlamydia and gonorrhoea among women in Victoria over the study period, from 47134 tests in 2008 to 110209 in 2013 (Box 1). The number of chlamydia tests alone increased 1.3-fold, from 56916 tests in 2008 to 76485 tests in 2013.

Notifications to the Victorian Department of Health of gonorrhoea cases identified by culture alone (excluding cases diagnosed at the MSHC) among women decreased slightly from 19 cases in 2008 to five cases in 2013. Gonorrhoea cases identified by culture alone and cases identified by NAAT and culture increased from 38 cases in 2008 to 86 cases in 2013. However, gonorrhoea cases identified by NAAT alone increased 3.5-fold, from 98 cases in 2008 to 343 cases in 2013 (Box 2). Of all NAATs performed in Victoria, the proportion of positive tests ranged between $0.2 \%$ and $0.3 \%$ and did not change over time ( $P$ for trend 0.66) (Box 3).

Over the same period, 35874 women were tested for gonorrhoea by culture alone at the MSHC, of whom $158(0.4 \%)$ tested positive. Gonococcal culture positivity among all women at the MSHC did not change over time ( $P$ for trend 0.70) (Box 3). Gonococcal culture positivity among sex workers was 3-4 times higher than among other heterosexual women, but the gonococcal culture positivity among sex workers did not change over time ( $P$ for trend 0.34 ). During 2010-2013, 25 women' were referred to the MSHC with a positive NAAT result for gonorrhoea and had not had any prior treatment, but only 10 of these women tested positive for gonorrhoea by culture alone at the MSHC, and nine of these presented with symptoms.

\section{Discussion}

Our study shows that most testing of gonorrhoea among women in Victoria is undertaken using NAAT detection, despite the very low population prevalence. Testing with dual NAAT rose 2.3-fold over the study period and was temporally associated with substantial increases in gonorrhoea notifications among women in Victoria. However, there was no observable increase in the proportion of women diagnosed with gonorrhoea by culture at the MSHC, or in the proportion of notifications per reported MBS item numbers for dual NAAT in Victoria. Taken collectively, these data suggest that the prevalence of gonorrhoea among women remains very low and stable in Victoria $(0.2 \%$ $0.3 \%)$ and at the MSHC (0.4\%-0.6\%), and that the rise in notifications is likely due to false-positive results arising from the increased use of NAATs in a lowprevalence population, ${ }^{6}$ with potential for unnecessary treatment of patients and partners and consequent harm.

These findings confirm current guidelines that testing for gonorrhoea with NAAT should be used when there is a clinical indication or for women at increased risk of gonorrhoea. ${ }^{10}$ We recommend that women being screened for chlamydia who are not at increased risk of gonorrhoea should be tested for chlamydia alone, or the gonorrhoea result supressed in the laboratory.

Our study has several limitations. First, the nature of notification data may be incomplete or inaccurate. However, in Victoria, notifications are received from both clinicians and laboratories, with duplicates carefully removed. Second, a small proportion of laboratory testing for gonorrhoea is done in public hospitals that do not use the MBS, so we may have overestimated 
the prevalence, but a lower prevalence would only strengthen our conclusions. Third, the sample of women referred to the MSHC for treatment of gonorrhoea who were initially diagnosed by NAAT was small, which may limit the statistical power. However, if these were real cases of gonorrhoea, one would expect about $90 \%$ to test positive by endocervical culture regardless of bias, but only $40 \%$ of untreated infection was confirmed by culture, suggesting that the remainder (60\%) were false positives. ${ }^{2,16}$ Finally, we do not know how many laboratories suppress gonorrhoea results from the dual NAAT. If a significant proportion do not supress gonorrhoea results when testing for chlamydia, the number of gonorrhoea notifications could have been overestimated, and up to $60 \%$ could be false positives based on our data.

Although endocervical culture is almost as sensitive as NAAT for that site, $^{2}$ only 10/25 of our NAAT-positive samples had positive endocervical cultures for gonorrhoea, suggesting that most of the positive gonorrhoea NAATs $(60 \%)$ are false positives. This finding is consistent with the product information and the screening recommendation from the US Preventive Services Task Force warning against routine screening for gonorrhoea with NAAT among individuals in a low-prevalence population (ie, $<1 \%)$. ${ }^{4,6}$

Previous studies have shown that the dual NAAT for chlamydia and gonorrhoea has high sensitivity and specificity, and does not require further confirmatory testing, ${ }^{17,18}$ however, this recommendation is only valid in high-prevalence populations. The Australian Public Health Laboratory Network guidelines recommend that cases identified by NAAT should be confirmed by supplementary testing in low-prevalence populations, or the PPV of the test should exceed $90 \%{ }^{19}$ A New Zealand study with low gonorrhoea prevalence $(0.77 \%)$ showed that the PPV of NAATs in female urogenital specimens reached $80 \%$ if a secondary confirmatory assay was not used..$^{20}$ Our lowest prevalence was about a third of this, so the PPV would be expected to be substantially lower. ${ }^{21}$

In the New Zealand study, when discordant results were evaluated by a clinical microbiologist, the PPV exceeded $90 \% .{ }^{20}$ Supplementary confirmatory testing is advocated in Australia, ${ }^{19,22}$ European countries ${ }^{23}$ and the United Kingdom. ${ }^{24}$ The proportion of Australian laboratories that do this is unknown.

Over our study period, there was a substantial increase in the proportion of laboratories using the dual NAAT for chlamydia and gonorrhoea. The Royal College of Pathologists of Australasia Molecular Quality Assurance Programs Diagnostics Report found that about $53 \%$ of participating laboratories used dual NAAT in 2013 compared with $8 \%$ in $2007.8,9$ This increase has been driven by the move to dual assays by the main manufacturers. The capacity to test for dual targets, with little or no difference in cost, may have contributed to this increase. If the gonorrhoea test is performed and is positive, the laboratory has a duty of care to report the positive gonorrhoea result. A recent study suggests that even when only chlamydia is ordered, most laboratories are reporting a positive gonorrhoea result. ${ }^{25}$ When this is done, the clinician needs to be aware of the possibility of a false positive, and a confirmatory test by culture should be performed. Ideally, laboratories should suppress any gonorrhoea result.

It is difficult to estimate the number of women, or low-risk heterosexual men, who receive a false-positive result from a gonorrhoea screening test. This number depends on the specificity of the assay used, and whether confirmatory testing is always carried out. The published specificity of the four most popular testing platforms varies from $98.8 \%$ to $99.9 \% .^{5,21,26,27}$ If $0.3 \%$ of NAATs ordered test positive, the PPV will be between $19 \%$ and $75 \%$ - a falsepositive rate between $25 \%$ and $81 \%$. If supplementary assays are always carried out, this will be substantially reduced. In a recent study in a New Zealand population with a prevalence of $0.77 \%, 22 \%(8 / 37)$ of NAAT-positive, culture-negative samples were evaluated by a clinical microbiologist and deemed to be false-positive results. ${ }^{20}$

Finding the balance between underand overdiagnosis of gonorrhoea among women at low risk is a difficult clinical and public health question. Currently, we are likely testing too many low-risk women, who are

\section{Number of women tested and positivity for gonorrhoea by culture at MSHC, and notifications by NAAT,* 2008-2013}

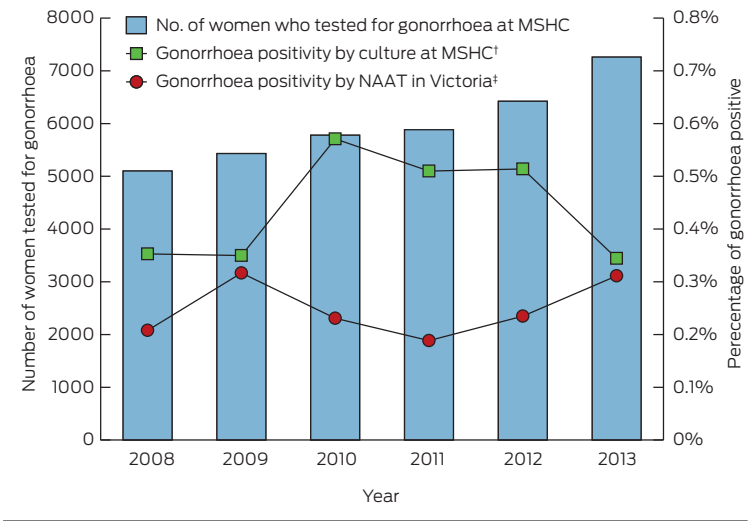

MSHC = Melbourne Sexual Health Centre. NAAT = nucleic acid amplification test. *As a proportion of reports of item numbers 69317 and 69319. $\uparrow P$ for trend, 0.70. $\ddagger P$ for trend, 0.66.

presumably attending a GP for chlamydia screening. A false-positive diagnosis of an STI has important implications for relationships, ${ }^{6}$ and unnecessary treatment may be associated with side effects. ${ }^{28}$

In conclusion, testing for gonorrhoea using NAAT is currently being done in a very low-prevalence population in Victoria, contrary to the manufacturers' and other guidelines, and likely resulting in a substantial number of false-positive results and unnecessary treatment. The current increase in notifications may be an artefact of more testing for gonorrhoea using automated multiplex NAAT. In Victoria, and Australia outside of high-prevalence populations (eg, Indigenous populations and MSM), ${ }^{12,29}$ testing should be minimised or done by culture unless the pretest probability is high (eg, symptoms of pelvic inflammatory disease, had a gonorrhoea contact, engaged in commercial sex, or had sex overseas). Positive gonococcal NAATs among low-risk women should then be confirmed by culture or regarded as doubtful. This will minimise the number of false-positive notifications, and reduce potential harm to individuals.

Acknowledgements: We acknowledge A Afrizal for his assistance with data extraction. This work was supported by the National Health and Medical Research Council (grant 568971).

Competing interests: No relevant disclosures.

Received 29 May 2014, accepted 17 Oct 2014.

References are available online at www.mja.com.au. 
1 Victorian Government Department of Health. Communicable disease surveillance, Victoria, April-June 2013. Victorian Infectious Diseases Bulletin 2013; 16: 113-131. http://docs.health.vic.gov.au/ docs/doc/B849617CC7EF06EICA257CF A0081AFB8/\$FILE/VIDB-16-3-web.pdf (accessed Oct 2014).

2 Johnson RE, Newhall WJ, Papp JR, et al. Screening tests to detect Chlamydia trachomatis and Neisseria gonorrhoeae infections - 2002. MMWR Recomm Rep 2002; 51: 1-38.

3 Katz AR, Effler PV, Ohye RG, et al. Falsepositive gonorrhea test results with a nucleic acid amplification test: the impact of low prevalence on positive predictive value. Clin Infect Dis 2004; 38 : 814-819.

4 Fifer $\mathrm{H}$, Ison CA. Nucleic acid amplification tests for the diagnosis of Neisseria gonorrhoeae in low-prevalence settings: a review of the evidence. Sex Transm Infect 2014; Jul 10. [Epub ahead of print]. doi: sextrans-2014-051588.

5 BD. BD ProbeTec ${ }^{\mathrm{TM}}$ ET Chlamydia trachomatis and Neisseria gonorrhoeae amplified DNA assays for use on the BD Viper'M System [package insert]. http:// www.bd.com/ds/technicalcenter/clsi/ clsi-viper-ctgc.pdf (accessed Oct 2014).

6 US Preventive Services Task Force. Screening for gonorrhea: recommendation statement. Ann Fam Med 2005; 3: 263-267.

7 Van Dyck E, leven M, Pattyn S, et al. Detection of Chlamydia trachomatis and Neisseria gonorrhoeae by enzyme immunoassay, culture, and three nucleic acid amplification tests. J Clin Microbiol 2001; 39: 1751-1756.

8 Royal College of Pathologists of Australasia Quality Assurance Programs. Summary report for Neisseria gonorrhoeae nucleic acid detection series (surveys 2007-2 and 2007-4). Sydney: RCPAQAP, 2007.

9 Royal College of Pathologists of Australasia Quality Assurance Programs. Summary report for Neisseria gonorrhoeae nucleic acid detection (survey 2013-5). Contract No. 2013: 5: 9A-F. Sydney: RCPAQAP, 2013.

10 Royal Australian College of General Practitioners. Guidelines for preventive activities in general practice. 8th ed. Melbourne: RACGP, 2012. http://www. racgp.org.au/download/Documents/
Guidelines/Redbook8/redbook8.pdf (accessed Oct 2014).

11 McDonagh P, Ryder N, McNulty AM, Freedman E. Neisseria gonorrhoeae infection in urban Sydney women: prevalence and predictors. Sex Health 2009; 6: 241-244.

12 Guy R, Garton L, Taylor-Thompson D, et al, editors. The 2010 baseline prevalence study conducted by the STRIVE trial. Australasian Sexual Health Conference; 2011 Sep 26-28; Canberra, Australia.

13 Chow EP, Fehler G, Chen MY, et al. Testing commercial sex workers for sexually transmitted infections in Victoria, Australia: an evaluation of the impact of reducing the frequency of testing. PLOS One 2014; 9: el03081.

14 Grimes DA, Schulz KF. Uses and abuses of screening tests. Lancet 2002; 359 : 881-884.

15 Australian Government Department of Health. MBS online: Medicare Benefits Schedule. http://www.mbsonline.gov. au/internet/mbsonline/publishing.nsf/ Content/Home (accessed Oct 2014).

16 Centers for Disease Control and Prevention. Recommendations for the laboratory-based detection of Chlamydia trachomatis and Neisseria gonorrhoeae 2014. MMWR Recomm Rep 2014; 63: 1-19.

17 Perry MD, Jones RN, Corden SA. Is confirmatory testing of Roche cobas 4800 CT/NG test Neisseria gonorrhoeae positive samples required? Comparison of the Roche cobas 4800 CT/NG test with an opa/pap duplex assay for the detection of N gonorrhoeae. Sex Transm Infect 2014; 90: 303-308.

18 Rockett R, Goire N, Limnios A, et al. Evaluation of the cobas 4800 CT/NG test for detecting Chlamydia trachomatis and Neisseria gonorrhoeae. Sex Transm Infect 2010; 86: 470-473.

19 Smith DW, Tapsall JW, Lum G. Guidelines for the use and interpretation of nucleic acid detection tests for Neisseria gonorrhoeae in Australia: a position paper on behalf of the Public Health Laboratory Network. Commun Dis Intell Q Rep 2005; 29: 358-365.

20 Bromhead C, Miller A, Jones M, Whiley D. Comparison of the cobas 4800 CT/NG test with culture for detecting Neisseria gonorrhoeae in genital and nongenital specimens in a low-prevalence population in New Zealand. J Clin Microbiol 2013; 51: 1505-1509.

21 Gaydos CA, Cartwright CP, Colaninno P, et al. Performance of the Abbott RealTime CT/NG for detection of Chlamydia trachomatis and Neisseria gonorrhoeae. J Clin Microbiol 2010; 48: 3236-3243.

22 Tabrizi SN, Hjelmevoll SO, Garland SM, Unemo M. Reply to "Routine confirmation of positive nucleic acid amplification test results for Neisseria gonorrhoeae is not necessary". J Clin Microbiol 2012; 50 : 209-210. doi:10.1128/JCM.06089-11.

23 Bignell C; IUSTI/WHO. 2009 European (IUSTI/WHO) guideline on the diagnosis and treatment of gonorrhoea in adults. Int J STD AIDS 2009; 20: 453-457.

24 Bignell C, Fitzgerald M; Guideline Development Group; British Association for Sexual Health and HIV UK. UK national guideline for the management of gonorrhoea in adults, 2011. Int J STD AIDS 2011; 22: 541-547.

25 Donovan B, Dimech W, Ali H, et al. Increased testing for Neisseria gonorrhoeae with duplex nucleic acid amplification tests in Australia: implications for surveillance. Sex Health 2015; Feb 9. [Epub ahead of print]. doi: 10.1071/SHI4179.

26 Gaydos CA, Quinn TC, Willis D, et al. Performance of the APTIMA Combo 2 assay for detection of Chlamydia trachomatis and Neisseria gonorrhoeae in female urine and endocervical swab specimens. J Clin Microbiol 2003; 41: 304-309.

27 Van Der Pol B, Liesenfeld O, Williams JA, et al. Performance of the cobas CT/ NG test compared to the Aptima AC2 and Viper CTQ/GCQ assays for detection of Chlamydia trachomatis and Neisseria gonorrhoeae. J Clin Microbiol 2012; 50 : 2244-2249.

28 Behets FM, Miller WC, Cohen MS. Syndromic treatment of gonococcal and chlamydial infections in women seeking primary care for the genital discharge syndrome: decision-making. Bull World Health Organ 2001; 79: 1070-1075.

29 Chow EPF, Tomnay J, Fehler G, et al. Substantial increases in chlamydia and gonorrhea positivity unexplained by changes in individual-level sexual behaviors among men who have sex with men in an Australian sexual health service from 2007-2013. Sex Transm Dis 2015; 42: 81-87. 